\title{
Epidemic Dynamics on an Adaptive Network
}

\author{
Thilo Gross, Carlos J. Dommar D’Lima, and Bernd Blasius \\ AG Nichtlineare Dynamik, Institut für Physik, Universität Potsdam, Am Neuen Palais 10, 14469 Potsdam, Germany
}

(Received 18 January 2006; published 24 May 2006)

\begin{abstract}
Many real-world networks are characterized by adaptive changes in their topology depending on the state of their nodes. Here we study epidemic dynamics on an adaptive network, where the susceptibles are able to avoid contact with the infected by rewiring their network connections. This gives rise to assortative degree correlation, oscillations, hysteresis, and first order transitions. We propose a low-dimensional model to describe the system and present a full local bifurcation analysis. Our results indicate that the interplay between dynamics and topology can have important consequences for the spreading of infectious diseases and related applications.
\end{abstract}

DOI: 10.1103/PhysRevLett.96.208701

PACS numbers: 89.75.Hc, 87.19.Xx, 89.75.Fb

In the physical literature the dynamics of complex networks has recently received much attention, with many applications in social, biological, and technical systems $[1,2]$. In particular, most research has been directed in two distinct directions. On the one hand, attention has been paid to the structure of the networks, revealing that simple dynamical rules, such as preferential attachment or selective rewiring, can be used to generate complex topologies [3-6]. Many of these rules are not only a useful tool for the generation of model graphs, but are also believed to shape real-world networks like the internet or the network of social contacts. On the other hand, research has focused on large ensembles of dynamical systems, where the interaction between individual units is described by a complex graph [7-15]. These studies have shown that the network topology can have a strong impact on the dynamics of the nodes, e.g., the absence of epidemic thresholds on scale free networks $[7,8]$ or the detrimental effect of assortative degree correlations on targeted vaccination [12]. In the past the cross fertilization between these two lines of thought has led to considerable advances. However, the dynamics of networks and the dynamics on networks are still generally studied separately. In doing so, a characteristic features of many real-world networks is not taken into account, namely, the ability to adapt the network topology dynamically in response to the dynamic state of nodes [16-19].

Consider, for example, the spreading of an infectious disease on a social network. Humans tend to respond to the emergence of an epidemic by avoiding contacts with infected individuals. Such rewiring of the local connections can have a strong effect on the dynamics of the disease, which in turn influences the rewiring process. Thus, a complicated mutual interaction between a time varying network topology and the dynamics of the nodes emerges.

In this Letter we study a susceptible-infectedsusceptible (SIS) model on an adaptive network. We demonstrate that a simple intuitive rewiring rule for the network connections has a profound impact on the emerging network, and is able to generate specific network properties such as a wide degree distribution, assortative degree correlations, and the formation of two loosely connected subcompartments. The dynamical consequences are the emergence of new epidemic thresholds (corresponding to first order transitions), the coexistence of multiple stable equilibria (leading to hysteresis), and the appearance of an oscillatory regime, all of which are absent on static SIS networks.

We consider a network with a constant number of nodes, $N$, and bidirectional links, $K$. The nodes represent individuals, which are either susceptible (S) or infected (I). In every time step and for every link connecting an infected with a susceptible (SI link), the susceptible becomes infected with the fixed probability $p$. The Infected recovers from the disease with probability $r$, becoming susceptible again. In addition, we allow susceptible individuals to protect themselves by rewiring their links. With probability $w$ for every SI link, the susceptible breaks the link to the infected and forms a new link to another randomly selected susceptible. Double connections and self-connections are not allowed to form in this way.

To study the effect of adaptive rewiring consider the threshold infection probability $p^{*}$ that is necessary to maintain a stable epidemic. On a random graph without rewiring $(w=0)$ the basic reproductive number, which denotes the secondary infections caused by a single infected node on an otherwise susceptible network is $R_{0}=$ $p\langle k\rangle / r$, where $\langle k\rangle=2 K / N$ is the mean degree of the nodes [20]. Demanding that exactly one secondary infection is caused yields $p^{*}=r /\langle k\rangle$. If rewiring is taken into account a single infected node will on average lose a constant fraction $w$ of its links. Therefore the degree of such a node can be written as $k(t)=\langle k\rangle \exp (-w t)$, where $t$ is the time since infection. By averaging over the typical lifetime $1 / r$ of an infected node, we obtain the threshold infection rate

$$
p^{*}=\frac{w}{\langle k\rangle[1-\exp (-w / r)]} .
$$

Note that this corresponds to $p^{*}=r /\langle k\rangle$ for $w=0$, but 
$p^{*}=w /\langle k\rangle$ for $w \gg r$. Thus, a high rewiring rate can significantly increase the epidemic threshold and thereby reduce the prevalence of the epidemic.

In comparison, the effect of adaptive rewiring on the topology is more subtle. Let us first consider the trivial case in which rewiring is independent of the state of the nodes (Fig. 1, left). In this case the degree distribution becomes Poissonian and the average degree $\left\langle k_{n n}\right\rangle$ of the next neighbors of a given node is independent of the degree $k$, as one would expect in a static random graph.

Now, assume that the adaptive rewiring rule described above is used, but the local dynamics is switched off, $r=$ $p=0$, (Fig. 1, center). In this case the density of the infected, $i$, and the susceptibles, $s=1-i$, stays constant [21]. However, the number of SI links is reduced systematically over time until the network has split into two disconnected clusters, one of which is occupied by the infected while the other is occupied by the susceptibles. Assuming that we start with a random graph, the per capita densities of SS, II, and SI links are initially $l_{\mathrm{SS}}=s^{2}\langle k\rangle / 2$, $l_{\mathrm{II}}=i^{2}\langle k\rangle / 2$, and $l_{\mathrm{SI}}=\langle k\rangle / 2-l_{\mathrm{SS}}-l_{\mathrm{II}}=s i\langle k\rangle$, respectively. With adaptive rewiring, in the stationary state all SI links have been converted into SS links so that $l_{\mathrm{SS}}=(1-$ $\left.i^{2}\right)\langle k\rangle / 2$ and $l_{\mathrm{SI}}=0$. Consequently, the susceptibles and the infected assume different degree distributions $\rho_{k}$, in which the mean degree of a susceptible node is $\left\langle k_{\mathrm{S}}\right\rangle=$ $(1+i)\langle k\rangle$ and the mean degree of an infected node is $\left\langle k_{\mathrm{I}}\right\rangle=i\langle k\rangle$. While both clusters are still individually

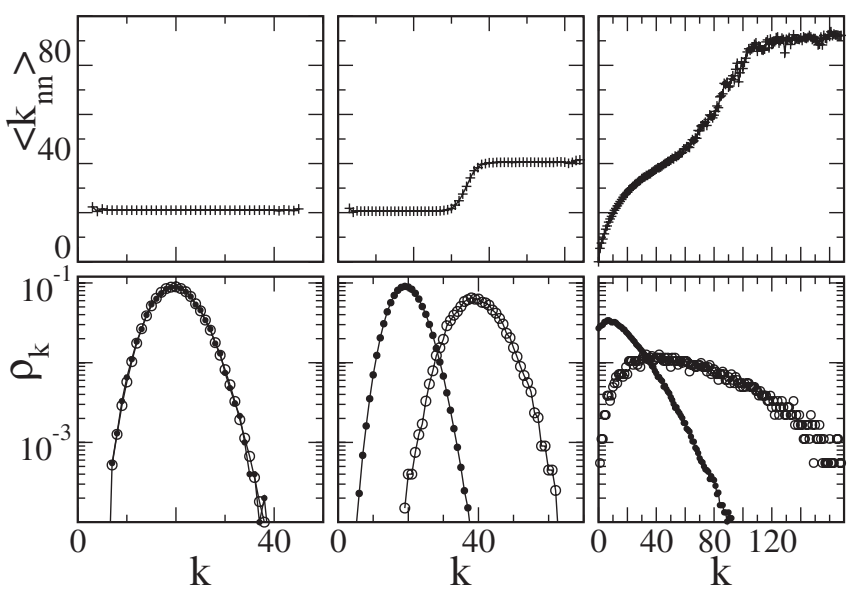

FIG. 1. Structure of adaptive networks. Plotted is the mean nearest-neighbor degree $\left\langle k_{n n}\right\rangle$ (top) and the degree distribution $\rho_{k}$ for susceptibles (bottom, circles) and infected (bottom, dots) depending on the degree $k$. (Left) Indiscriminate rewiring: the network is a random graph with Poissonian degree distributions and vanishing degree correlation. (Center) No local dynamics $(p=r=0)$ : the infected and the susceptibles separate into two unconnected random subgraphs. (Right) Adaptive network with rewiring and local dynamics $(w=0.3, r=0.002, p=0.008)$ : the degree distributions are broadened considerably and a strong assortative degree correlation appears. The plots correspond to $N=10^{5}, K=10^{6}$.
Poissonian, the susceptible cluster has a higher connectivity. Since $\left\langle k_{\mathrm{nn}}\right\rangle$ is independent of $k$ in each of the two clusters, the degree correlation within the cluster vanishes. However, a considerable net degree correlation $r_{\text {corr }}>0$ [22] can arise if both clusters are considered together because $\left\langle k_{n n}\right\rangle$ is larger for the susceptible cluster.

Finally, consider the case with both adaptive rewiring and epidemic dynamics (Fig. 1, right). Even though rewiring is not fast enough to separate infected and susceptible completely, it still structures the system into two loosely connected clusters of susceptibles and infected (e.g., $l_{\mathrm{SI}} \approx$ $0.01\langle k\rangle$ in the figure). While intercluster connections are continuously removed by rewiring, new ones are formed by recoveries in the infected cluster and infections in the susceptible cluster. This leads to large temporal fluctuations in the degree of a node. As long as an individual is susceptible, its degree is increasing approximately linear in time, $\dot{k}=w l_{\mathrm{SI}}$, due to the rewiring activity of the other susceptibles. In contrast, the degree of infected decays exponentially, $\dot{k} \sim-w k$. In this way, a complicated dynamical equilibrium can form in which the average number of intercluster and intracluster links as well as the density of susceptibles and infected stays constant. In this equilibrium the continuous rewiring of connections leads to broadened degree distributions for both infected and susceptibles and a positive (assortative) degree correlation.

The effect of adaptive rewiring on the emerging network structure is further quantified in Fig. 2. With increasing $w$ the degree correlation grows rapidly. Moreover, the mean degree of the susceptibles increases while the degree of the infected decreases slightly. Even more pronounced is the increase in the variance of the degree distribution of the susceptibles, e.g., for $w=0.6$ the variance $\sigma^{2}$ rises by

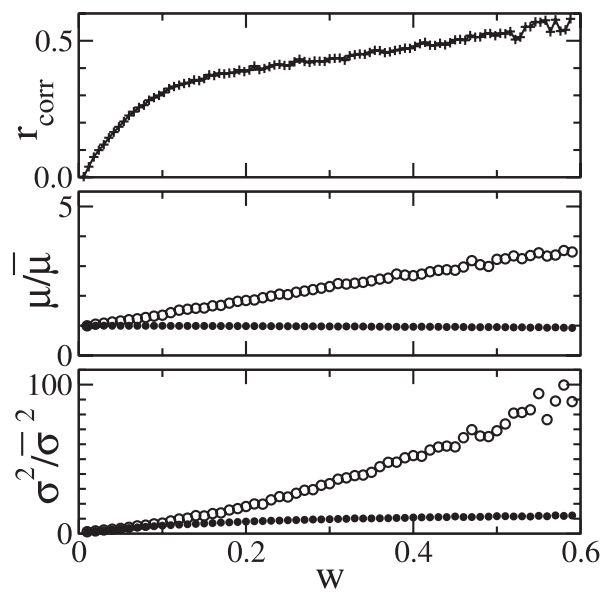

FIG. 2. Degree correlation index $r_{\text {corr }}$ [22] as a function of the rewiring rate (top). Furthermore, the mean $\mu$ (center) and the variance $\sigma^{2}$ (bottom) of the degree distributions for the susceptibles (circles) and the infected (dots) are shown. Both quantities have been normalized with respect to their values in a random graph without rewiring, $\bar{\mu}$ and $\bar{\sigma}^{2}$, respectively. The plots correspond to $N=10^{5}, K=10^{6}, r=0.002, p=0.008$. 
a factor of 100 in Fig. 2. This indicates the formation of strongly connected hubs and temporarily isolated nodes, which are rapidly reconnected because of rewiring.

As we have shown, adaptive rewiring promotes the isolation of infected individuals, which can significantly increase the epidemic threshold. However, in doing so rewiring introduces a mixing of connections in the population and also leads to the formation of a highly connected susceptible cluster, which is characterized by a large variance of the degree distribution and hence has a lower epidemic threshold. Therefore the local effect of rewiring tends to suppress the epidemic while the topological effect promotes it. In order to investigate the dynamics caused by the opposing effects of rewiring it is useful to consider a low-dimensional model. From the discussion above both the dynamic state and the topological structure of the network can be described in terms of the mean field quantities $i$ and $l_{\mathrm{SS}}$ and $l_{\mathrm{II}}$. To describe the time evolution of these variables we apply the moment closure approximation proposed in Ref. [23]. In this pair approximation the density of all triples $l_{a b c}$ in the network with the respective states $a, b, c \in[\mathrm{S}, \mathrm{I}]$ are approximated by $l_{a b c}=l_{a b} l_{b c} / b$, i.e., as the product of the number of $a b$ links $l_{a b}$ and the probability $l_{b c} / b$ that a given node of type $b$ has a $b c$ link. This leads to a system of three coupled ordinary differential equations

$$
\begin{gathered}
\frac{d}{d t} i=p l_{\mathrm{SI}}-r i, \\
\frac{d}{d t} l_{\mathrm{II}}=p l_{\mathrm{SI}}\left(\frac{l_{\mathrm{SI}}}{s}+1\right)-2 r l_{\mathrm{II}} \\
\frac{d}{d t} l_{\mathrm{SS}}=(r+w) l_{\mathrm{SI}}-\frac{2 p l_{\mathrm{SI}} l_{\mathrm{SS}}}{s} .
\end{gathered}
$$

The first term in Eq. (2) describes the infection of susceptible individuals, while the second term describes recovery. These two processes also effect the dynamics of the links. The first term in Eq. (3) corresponds to the conversion of SI links into II links as a result of new infections while the second term represents the conversion of II links into SI links as a result of recovery. Equation (4) is analogous except that the conversion of SI links into SS links by rewiring has been taken into account.

In Fig. 3 the analytical results from the low order model are compared with direct numerical simulations of the full model. Without rewiring, there is only a single, continuous dynamical transition, which occurs at the well-known epidemic threshold $p^{*}$. As the rewiring is switched on, this threshold increases in perfect agreement with Eq. (1). While the epidemic threshold still marks the critical parameter value for invasion of new diseases another, lower threshold, corresponding to a saddle-node bifurcation, appears. Above this threshold an already established epidemic can persist (endemic state). In contrast to the case without rewiring the two thresholds correspond to discontinuous (first order) transitions. Between them a region of bistability is located, in which the healthy and endemic state are both stable. Thus, a hysteresis loop is formed.

Our numerical simulations show that the presence of a hysteresis loop and first order transitions is a generic feature of the adaptive model and can be observed at all finite rewiring rates. While increasing the rewiring rate hardly reduces the size of the epidemic in the endemic state, the nature of the persistence threshold changes at higher rewiring rates. First, a subcritical Hopf bifurcation, which gives rise to an unstable limit cycle replaces the saddle-node bifurcation. At even higher rewiring rates this Hopf bifurcation becomes supercritical. Since the emerging limit cycle is now stable, the Hopf bifurcation marks a third threshold at which a continuous transition to oscillatory dynamics occurs. However, these oscillations can only be observed in a relatively small parameter region (see Figure 4) before the persistence threshold is encoun-
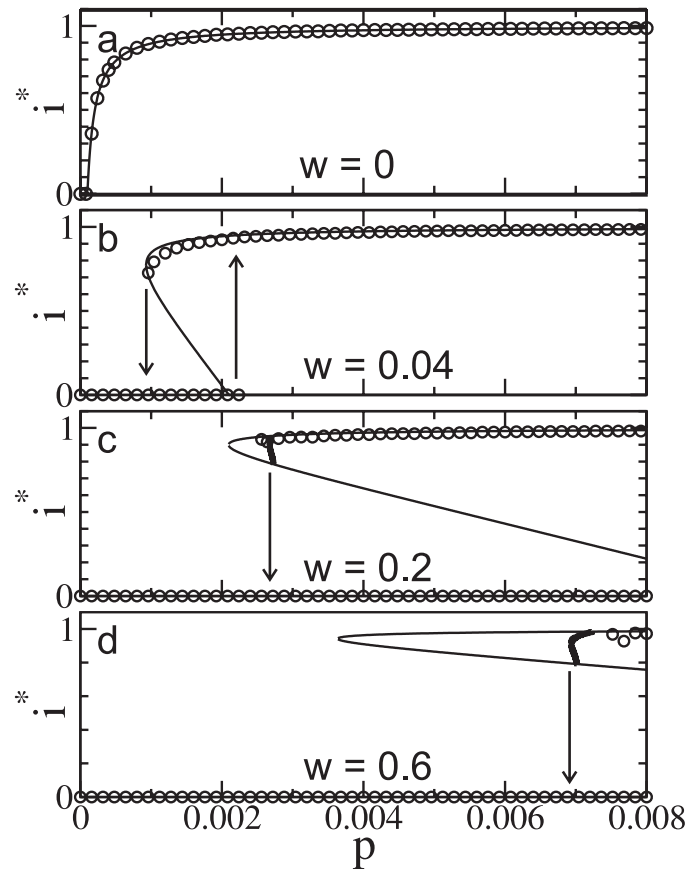

FIG. 3. Bifurcation diagram of the density of the infected $i^{*}$ as a function of the infection probability $p$ for different values of the rewiring rate $w$. In each diagram $i^{*}$ has been computed analytically from Eqs. (2)-(4) (thin lines). Along the stable branches these results have been confirmed by the explicit simulation of the full network (circles). Without rewiring only a single continuous transition occurs at $p^{*}=0.0001$ (a). By contrast, with rewiring a number of discontinuous transitions, bistability, and hysteresis loops (indicated by arrows) are observed (b), (c), (d). Fast rewiring (c), (d) leads to the emergence of limit cycles (thick lines indicate the lower turning point of the cycles), which have been computed numerically with the bifurcation software AUTO [24]. Parameter values $N=10^{5}, K=$ $10^{6}$, and $r=0.002$. 


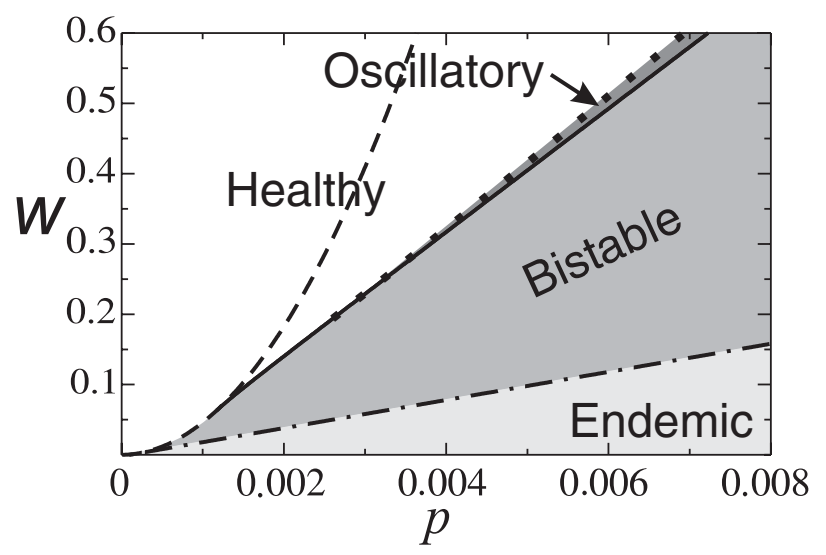

FIG. 4. Two parameter bifurcation diagram showing the dependence on the rewiring rate $w$ and the infection probability $p$. In the white and light gray regions there is only a single attractor, which is a healthy state in the white region and an endemic state in the light gray region. In the medium gray region both of these states are stable. Another smaller region of bistability is shown in dark gray. Here, a stable healthy state coexists with a stable epidemic cycle. The transition lines between these regions correspond to transcritical (dash-dotted), saddle-node (dashed), Hopf (continuous), and cycle fold (dotted) bifurcations. The transcritical bifurcation line agrees very well with Eq. (1). Note that the saddle-node and transcritical bifurcation lines emerge from a cusp bifurcation at $p=0.0001, w=0$. Parameters are as in Fig. 3.

tered, which now corresponds to a fold bifurcation of cycles.

In summary, we have shown that the interplay between the dynamics and topology of an adaptive network can give rise to rich dynamics. Here we have studied only the simplest example of an adaptive network, in which the number of nodes and links remains constant and the local dynamics is simple. Nevertheless, the adaptive nature of the system gives rise to dynamical features, like bistability and cycles. While we observe stable oscillations only in a small parameter region, the fact that they already appear in this simple example indicate that they can also be expected in more complex models. In fact, we have found much larger oscillatory regions in other model variants with different rewiring rules. Besides epidemic dynamics our findings also have strong implications for the spreading of information, opinions and beliefs in a population, which can be described in a similar way.

For the control of real-world diseases adaptive rewiring is beneficial since it increases the invasion threshold and also the persistence threshold for epidemics. However, the topological changes that are induced as a natural response to an emerging disease are cause for concern. The topology at the peak of a major epidemic can be very different from that in the disease-free state. In particular, positive degree correlations can rapidly arise, reducing effectiveness of targeted vaccination. Further, the formation of a densely linked cluster of susceptibles at high infection densities can enable the persistence of diseases which would not be able to persist at low infection densities. Therefore, a disease which seems to be a minor problem while it is rare can be very difficult to combat once it has reached an endemic state.

This work was supported by the Volkswagen-Stiftung.

[1] R. Albert and A.-L. Barabási, Rev. Mod. Phys. 74, 47 (2002).

[2] M.E. J. Newman, SIAM Rev. 45, 167 (2003).

[3] D. J. Price, Science 149, 510 (1965).

[4] D. J. Watts and S. H. Strogatz, Nature (London) 393, 440 (1998).

[5] A.-L. Barabási and R. Albert, Science 286, 509 (1999).

[6] S. N. Dorogovtsev, J.F. F. Mendes, and A. N. Samukhin, Phys. Rev. Lett. 85, 4633 (2000).

[7] R. Pastor-Satorras and A. Vespignani, Phys. Rev. Lett. 86, 3200 (2001).

[8] R. M. May and A.L. Lloyd, Phys. Rev. E 64, 066112 (2001).

[9] M. Kuperman and G. Abramson, Phys. Rev. Lett. 86, 2909 (2001).

[10] M. Barahona and L. M. Pecora, Phys. Rev. Lett. 89, 054101 (2002).

[11] V. M. Eguiluz and K. Klemm, Phys. Rev. Lett. 89, 108701 (2002).

[12] M.E. J. Newman, Phys. Rev. Lett. 89, 208701 (2002).

[13] M. Boguñá, R. Pastor-Satorras, and A. Vespignani, Phys. Rev. Lett. 90, 028701 (2003).

[14] M.E. J. Newman, Phys. Rev. E 67, 026126 (2003).

[15] R. Xulvi-Brunet and I. M. Sokolov, Phys. Rev. E 70, 066102 (2004).

[16] S. Bornholdt and T. Rohlf, Phys. Rev. Lett. 84, 6114 (2000).

[17] C. Zhou and J. Kurths, Phys. Rev. Lett. (to be published).

[18] H. Ebel and S. Bornholdt, cond-mat/0211666.

[19] P. Holme and G. Ghoshal, Phys. Rev. Lett. 96, 098701 (2006).

[20] R. M. Anderson and R. M. May, Infectious Diseases of Humans (Oxford University Press, Oxford, 1992).

[21] We use upper (lower) case letters to denote the state (density) of a node.

[22] In undirected networks the degree correlation can be computed as $r_{\text {corr }}=\sigma_{q}^{-2} \sum_{a b} a b\left(e_{a b}-q_{a} q_{b}\right)$ where $q_{a}=$ $(a+1) \rho_{a+1} / \sum_{k} k \rho_{k}, e_{a b}$ is the probability that a randomly chosen link connects nodes with the degrees $a+$ 1 and $b+1$, and $\sigma_{q}^{2}$ is the variance of the distribution $q_{a}$ [12].

[23] M. J. Keeling, D. A. Rand, and A. J. Morris, Proc. R. Soc. B 264, 1149 (1997); M. J. Keeling and K. T. D. Eames, J. R. Soc. Interface 2, 295 (2005).

[24] E. J. Doedel, R. C. Paffenroth, A. R. Champneys, T. F. Fairgrieve, Yu. A. Kuznetsov, B. Sandstede, and X. Wang, Caltech Technical Report, 2001. 